\title{
Crisis Hipertensivas
}

\section{Sr. Editor:}

Las crisis hipertensivas $(\mathrm{CH})$ son una patología frecuente en nuestras consultas y servicios de urgencias. Se estima que 1-2 de cada 100 pacientes con hipertensión arterial (HTA) presentarán una crisis hipertensiva a lo largo de su vida, pudiendo llegar a poner en riesgo su vida ${ }^{1}$, por lo que su correcta identificación, enfoque y manejo terapéutico, es fundamental en nuestro medio diario.

Una crisis hipertensiva es un aumento agudo de la presión arterial (PA), que puede ocasionar alteraciones orgánicas o funcionales sobre órganos diana. Se ha establecido como punto de corte una presión arterial sistólica (PAS) $\geq 180 \mathrm{mmHg}$ y/o una presión arterial diastólica (PAD) $\geq 110 \mathrm{mmHg}^{1,2}$.

Una vez identificada la $\mathrm{CH}$, hemos de investigar si se trata de una urgencia o emergencia hipertensiva, dándonos la respuesta en el $95 \%$ de los casos una adecuada historia clínica ${ }^{3}$.

En la urgencia hipertensiva el incremento de la PA no se acompaña de lesión en órgano diana y el paciente puede estar asintomático o con síntomas inespecíficos, no comprometiendo la vida del paciente. El tratamiento será vía oral, con un descenso en la PA no rápido (se reducirá un $20 \%$ en las primeras 24-48 horas para evitar el riesgo de hipoperfusión) ${ }^{1}$, y ante una evolución favorable se dará el alta al paciente para ajuste del tratamiento hipertensivo por su médico de familia.

En la emergencia hipertensiva el aumento de la PA se acompaña de lesión de órganos diana, pudiendo poner en riesgo la vida del paciente, por lo que requiere un descenso rápido (vía parenteral) e ingreso hospitalario ${ }^{1}$.

Si nos centramos en el tratamiento de la urgencia hipertensiva, que es más prevalente en nuestro medio, existen algunas revisiones de guías clínicas que nos pueden aclarar nuestra actitud terapéutica ante la heterogeneidad de información sobre este tema. Algunos puntos más controvertidos son la vía de administración y los fármacos a utilizar.

Las vías de administración, oral y/o sublingual, no son similares y conllevan importantes diferencias.
Así, la vía sublingual no es aceptada por todas la guías por su absorción errática e impredicible, optando por la vía oral ${ }^{1,3}$. Sin embargo, llama la atención cómo manuales de urgencias, boletines editados recientemente o alguna guía clínica indican esta vía ${ }^{4,5,6}$.

Con respecto a algunos fármacos utilizados, hemos de dedicarle una especial mención al nifedipino. Se trata de un calcio-antagonista dihidropiridínico de acción corta (6 horas) que disminuye eficazmente la PA, pero con el riesgo de producir eventos isquémicos por su velocidad de descenso. También produce vasodilatación, y secundariamente un fenómeno de robo en lechos vasculares, con taquicardia refleja por descarga de catecolaminas ${ }^{1}$. En embarazadas no es teratogénico, pero puede reducir la PA demasiado rápido y retrasar el parto por reducir las contracciones uterinas, por lo que su uso se debe evitar ${ }^{4}$. El nifedipino es un fármaco ampliamente utilizado por los servicios de urgencias, cuyo uso es apoyado y rechazado por algunas guías clínicas ${ }^{1}$, y su utilización por vía sublingual debe plantearse con prudencia porque su riesgo/beneficio parece desfavorable. Se encuentra formalmente contraindicado en pacientes con infarto agudo de miocardio.

En resumen, conviene desestimar la vía sublingual por la oral, debiéndonos plantear la alternativa a fármacos como el nifedipino por otros con menos efectos secundarios y diferentes características farmacocinéticas que nos permiten un uso más seguro. El captopril es el fármaco de elección de las $\mathrm{CH}$. Otras opciones que consideran la mayoría de las guías clínicas son nicardipino (con una vida media más larga que el nifedipino) y labetalol. En una minoría de guías también se incluyen lacidipino $y$ atenolol ${ }^{1,2,3}$.

Para concluir, deseo expresar que, aunque el volumen de información sea numeroso y creciente en nuestra profesión, hemos de valorar las publicaciones con detalle y juiciosamente en base a otro tipo de información, por ejemplo revisiones sistemáticas, para poderlas integrar adecuadamente en nuestro conocimiento y práctica clínica diaria. 


\section{BIBLIOGRAFÍA}

1. Caldevilla Benardo D, Martínez Pérez J, Artigao Ródenas LM, Divisón Garrote JA, Carbayo González Herencia JA, Massó Orozco J. Crisis Hipertensivas. Rev Clin Med Fam. 2008; 2(5):236-43

2. Mancia G, Baker G, Dominiczak A, Cifkova R, Fajard R, Germano $\mathrm{G}$ et al. Guidelines for the Management of Arterial Hypertension of the European Society of Hypertension (ESH) and the European Society of Cardiology (ESC). J Hypertension. 2007; 25:1105-87.

3. Regidor D, Fernández MA, Roca A, Rodríquez L. Crisis Hipertensivas en Urgencias. En: Julián A.editor. Manual de Protocolos y Actuación en Urgencias. $3^{\mathrm{a}}$ ed. Toledo: Edicomplet-Grupo SANED; 2010. p. 301-8.

4. Zarca MT, García López F, Muñoz Contreras MI. Tratamiento farmacológico de las patologías prevalentes en el embarazo. Boletín Farmacoterapéutico de Castilla-La Mancha. 2010; 11(4):1-4.
5. Jiménez M, Millán C, Llamas L, Montero FJ. Urgencia Hipertensiva. En: Jiménez L, Montero FJ, editores. Medicina de Urgencias y emergencias: Guía Diagnóstica y Protocolos de Actuación. $4^{\text {a }}$ ed. Barcelona: Elsevier; 2010. p. 234-9.

6. Chayán ML, Gil J, Moliner JR, Ríos MT, Castiñeira MC, González C, et al. Crisis Hipertensivas. Guías clínicas. 2010. Disponible en: http://www.fisterra.com/fisterrae/ guias.asp

Isabel Ponce García. Médico de Familia Centro de Salud Casas Ibáñez Miembro del Grupo de Trabajo de HTA de SEMFYC

Ctra. Valencia $s / n$, 02200 - Casas Ibáñez (Albacete). Correo electrónico: isabelponce81@hotmail.com

\section{FE DE ERRATAS}

En el artículo "Carcinomatosis meníngea por invasión dural de metástasis craneal", del que son autores Juan Ignacio Ramos-Clemente Romero, Eduardo Durán Ferreras, Miguel Ángel Pérez Ramos y Juan Manuel Domínguez Herrera, publicado el 1 de octubre de 2010 (número 3, volumen 3), apareció repetida la figura 1 , en lugar de aparecer la figura 2 , la cual corresponde a la siguiente imagen:

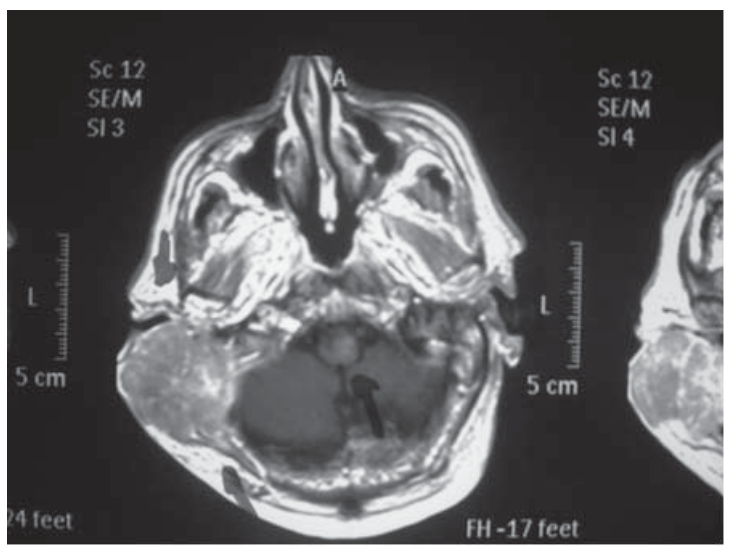

Figura 2. RM craneal. Corte axial en secuencia T1 que muestra masa tumoral en región auricular derecha que infiltra hueso temporal y occipital destruyendo la cortical y contactando con las meninges.

\section{CAMBIO DE DIRECCIÓN}

Le rogamos nos avise con antelación en caso de cambio de dirección para no alterar el servicio de recepción de REVISTA CLIINICA DE MEDICINA DE FAMILIA.

Los cambios deben comunicarse en el teléfono 967507911 o a través del correo electrónico info@scamfyc.org

\section{SUSCRIPCIONES Y ATENCIÓN AL CLIENTE}

\section{SCAMFYC}

Dionisio Guardiola, $16,4^{\circ} \mathrm{D}$

02003- Albacete

Teléfono y Fax: 967507911

Correo electrónico: info@scamfyc.org

Tarifa de suscripción anual

Profesionales 40,00 Euros (IVA incluido)

Instituciones 120,00 Euros (IVA incluido) 